\title{
Experimental and numerical modelling of the ventriculosinus shunt (El-Shafei shunt)
}

\author{
K Van Canneyt $^{1 * \dagger}$, J Kips ${ }^{1,2 \dagger}$, G Mareels ${ }^{1}$, E Baert ${ }^{3}$, D Van Roost ${ }^{3}$, and P Verdonck ${ }^{1}$ \\ ${ }^{1}$ Institute Biomedical Technology (IBiTech), Ghent University, Ghent, Belgium \\ ${ }^{2}$ Department of Pharmacology, Ghent University, Ghent, Belgium \\ ${ }^{3}$ Department of Neurosurgery, Ghent University Hospital, Ghent, Belgium
}

The manuscript was received on 29 January 2007 and was accepted after revision for publication on 1 October 2007.

DOI: 10.1243/09544119JEIM299

\begin{abstract}
This study assesses malresorptive hydrocephalus treatment by ventriculosinus shunting with the shunt in the antegrade or retrograde position. First, an experimental model of the cerebral ventricles, the arachnoid villi, the cortical veins, and the superior sagittal sinus was built. For this purpose, the compliance of a human cortical vein was measured and then modelled by means of Penrose tubes. The dimensions of the superior sagittal sinus were determined in vivo by measurements on magnetic resonance imaging scans of 21 patients. Second, a numerical model of the cortical veins and the superior sagittal sinus was built. The numerical results were validated with the results from the experimental model. The experimental and numerical pressure difference between the intracranial pressure and the static sinus pressure was small (0-20 Pa) and corresponded to the theoretically expected values. No overdrainage was found in either the antegrade or the retrograde position of the shunt. Blood reflow was only found while mimicking lumbar puncture or changes in position with the experimental model (lowering the intracranial pressure or increasing the sinus pressure rapidly). Optimal results can be obtained with the shunt positioned in the most downstream half of the superior sagittal sinus. The experimental and numerical results confirm the potential of ventriculosinus shunting as therapy for malresorptive hydrocephalus patients. The ventriculosinus shunt thus proves to be a promising technique.
\end{abstract}

Keywords: experimental, numerical, ventriculosinus shunt, hydrocephalus, overdrainage, superior sagittal sinus

\section{INTRODUCTION}

In normal physiological conditions, cerebrospinal fluid (CSF) is drained to the superior sagittal sinus (SSS) via small granulations, the arachnoid villi. Malresorptive hydrocephalus occurs when the arachnoid villi are unable to drain enough CSF, and the intracranial pressure (ICP) rises. Standard therapy includes draining the CSF by a shunt from the cerebral ventricles to the peritoneum (ventriculoperitoneal shunt) or the right atrium of the heart (ventriculoatrial shunt). However, both shunt types

*Corresponding author: Institute Biomedical Technology (IBiTech), Ghent University, De Pintelaan 185, Ghent, B-9000, Belgium.email: Koen.VanCanneyt@UGent.be

${ }^{\dagger}$ Both these authors contributed equally to this study are associated with major complications, one of them being siphonage [1-5]. This siphon effect, which develops when the patient assumes the erect position, can cause intraventricular hypotension and overdrainage [6].

A new type of shunt, the ventriculosinus (VS) shunt, was proposed by El-Shafei and El-Shafei [7]. Figure 1 shows the shunt connecting the cerebral ventricles to the SSS. Major advantages of this VS shunt are its short length and the use of an antisiphon effect caused by collapse of the internal jugular vein [8]. The VS shunt can be placed antegrade or retrogade (shunt tip with or against the direction of the SSS flow respectively). A potential drawback of all ventriculovenous (e.g. ventriculojugular and VS) shunts is (temporary) reflow. Reflow can be associated with blood stagnations, 


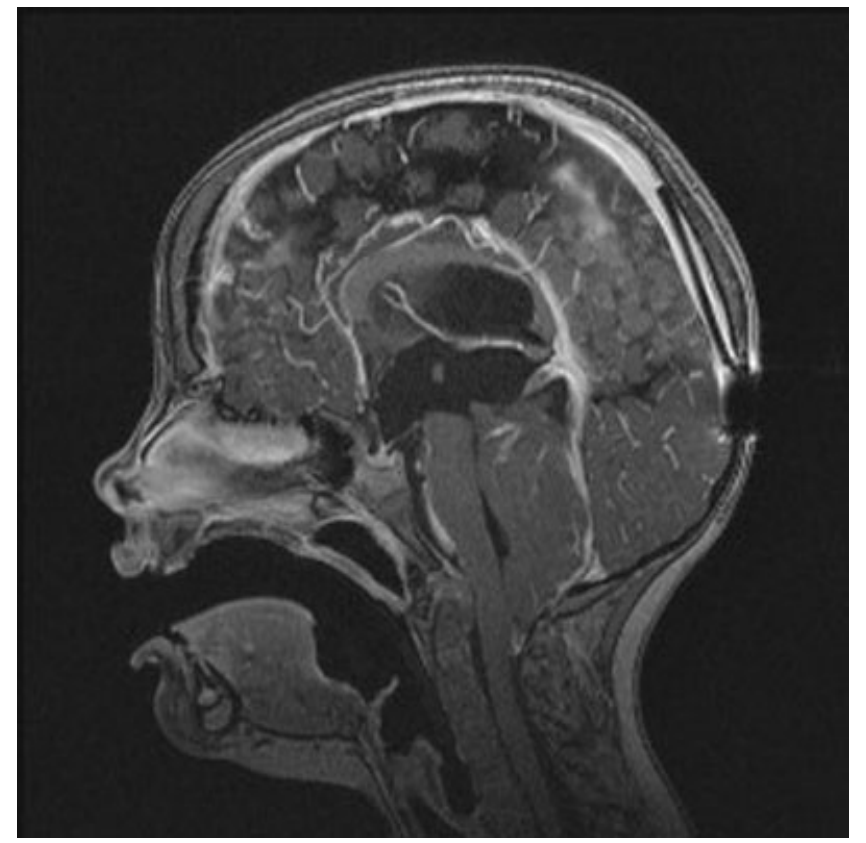

Fig. 1 Magnetic resonance imaging scan of an in-vivo VS shunt

thus triggering thrombus formation. This can obstruct the shunt and make it useless.

This study evaluates malresorptive hydrocephalus treatment by VS shunting. Overdrainage, reflow, and non-reflow conditions are assessed using an experimental and numerical model in order to have a detailed look at the flow and the pressures in the SSS, complementary to theoretical considerations and previous clinical studies.

The experimental model was constructed to study pressures on different locations associated with the insertion of a VS shunt in either the antegrade or the retrograde position. The ICP and the pressure in the SSS at the shunt tip were measured continuously. The transitional behaviour of the model towards steady state conditions and the steady state pressure difference between ICP and SSS pressure at the end of the shunt can be evaluated with the shunt in both the antegrade and the retrograde position to assess the shunt performance in clinical practice. In addition to this transitional behaviour, an extra test was included to investigate the impact of a change in the patient's position from upright to lying or to mimic a lumbar puncture.

A numerical model was developed to support the experimental findings. The steady state ICP and SSS pressure at the shunt tip were measured to assess reflow or overdrainage. Furthermore, a parametric study was included to investigate the impact of the shunt location in the SSS and a different flowrate on the correct functioning of the VS shunt.
Finally, both the experimental and the numerical results are compared with theoretical considerations and with the conclusions and clinical results of ElShafei and El-Shafei [7].

\section{MATERIAL AND METHODS}

\subsection{Experimental model}

The experimental set-up is shown in Fig. 2. It contains the human ventricles (A), the SSS (C), and the cerebral venous system (D). The human brain counts four cerebral ventricles, where the majority of CSF is produced and conserved. These ventricles are connected to the subarachnoidal space around the brain tissue and in the spinal cord. Both the ventricles and the subarachnoidal space are modelled as one CSF reservoir (Fig. 2, A), and so the reservoir pressure corresponds to the ICP and the CSF pressure. The total compliance of the CSF reservoir was set at $0.008 \mathrm{ml} /$ $\mathrm{Pa}$, corresponding to a pressure-volume index of $25 \mathrm{ml}$ at $10 \mathrm{mmHg}$ [9]. Compliance is set by use of a windkessel attached to the CSF reservoir (Fig. 2, B). CSF is modelled as pure water.

Since the shunt is inserted in the SSS, which has a triangular cross-section, it is important to model this SSS geometry accurately. The proximal upstream entering veins lead antegrade into the SSS and the most downstream veins lead retrograde into the SSS [10-12]. The SSS can be seen as incompressible [13, 14]. The length of the SSS and the surface area were measured along the SSS on magnetic resonance images of 21 patients (between 47 and 68 years old). The SSS surface areas were measured in three different cross-sectional planes. These planes were chosen at the end of the SSS (confluens sinuum), at the coronal suture, and in the middle between coronal suture and the beginning of the SSS. The beginning of the SSS was determined as the first point where the SSS was visible on magnetic resonance images. In the three planes, the base and height of the approximate triangular crosssections were measured and the corresponding surface area was calculated. Additionally, the exact surface area of the SSS at the chosen locations was measured. Figure 3 shows the measured and calculated values, from the beginning $(0 \mathrm{~cm})$ to the confluens sinuum $(28 \mathrm{~cm})$. As can be seen in Fig. 3, a geometry that fitted between the two values was used (an exception was made for the beginning of the SSS). The length of the measured SSS was $28.2 \pm$ $1.7 \mathrm{~cm}$. Consequently, a triangular profile was milled out of Plexiglas, $28 \mathrm{~cm}$ in length (Fig. 2, C, and Fig. 4). 


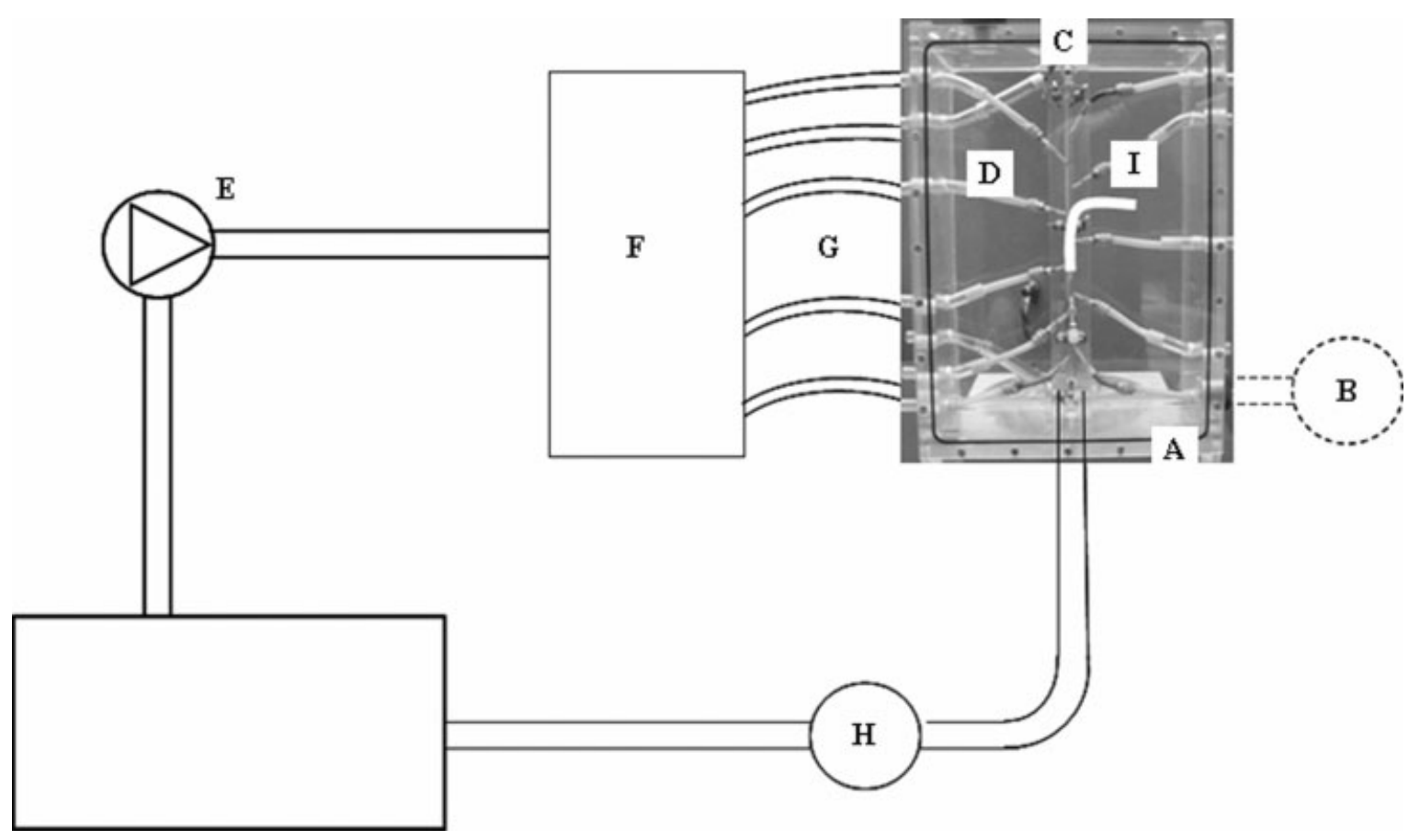

Fig. 2 Experimental set-up: A, CSF reservoir; B, windkessel; C, SSS; D, Penrose tubes modelling cortical veins; E, pump; F, distributor; G, rigid tubes; H, pressure reservoir; I, VS shunt

In order to model the cortical veins physiologically, two parameters are particularly important, namely the inflow velocity of the blood from the veins in the SSS and the compliance of the veins. About 40 cortical veins flow into the SSS, each with an average diameter of $1.5 \mathrm{~mm}$ [10]. In the model, they are represented by ten veins with a diameter of $3 \mathrm{~mm}$. This ensures that the inlet velocity in the SSS remains similar to the in-vivo situation. Second, a compliance measurement was performed on a human cortical vein (diameter, $1.5 \mathrm{~mm}$; length, $2 \mathrm{~cm}$ ) in order to quantify the magnitude of the compliance

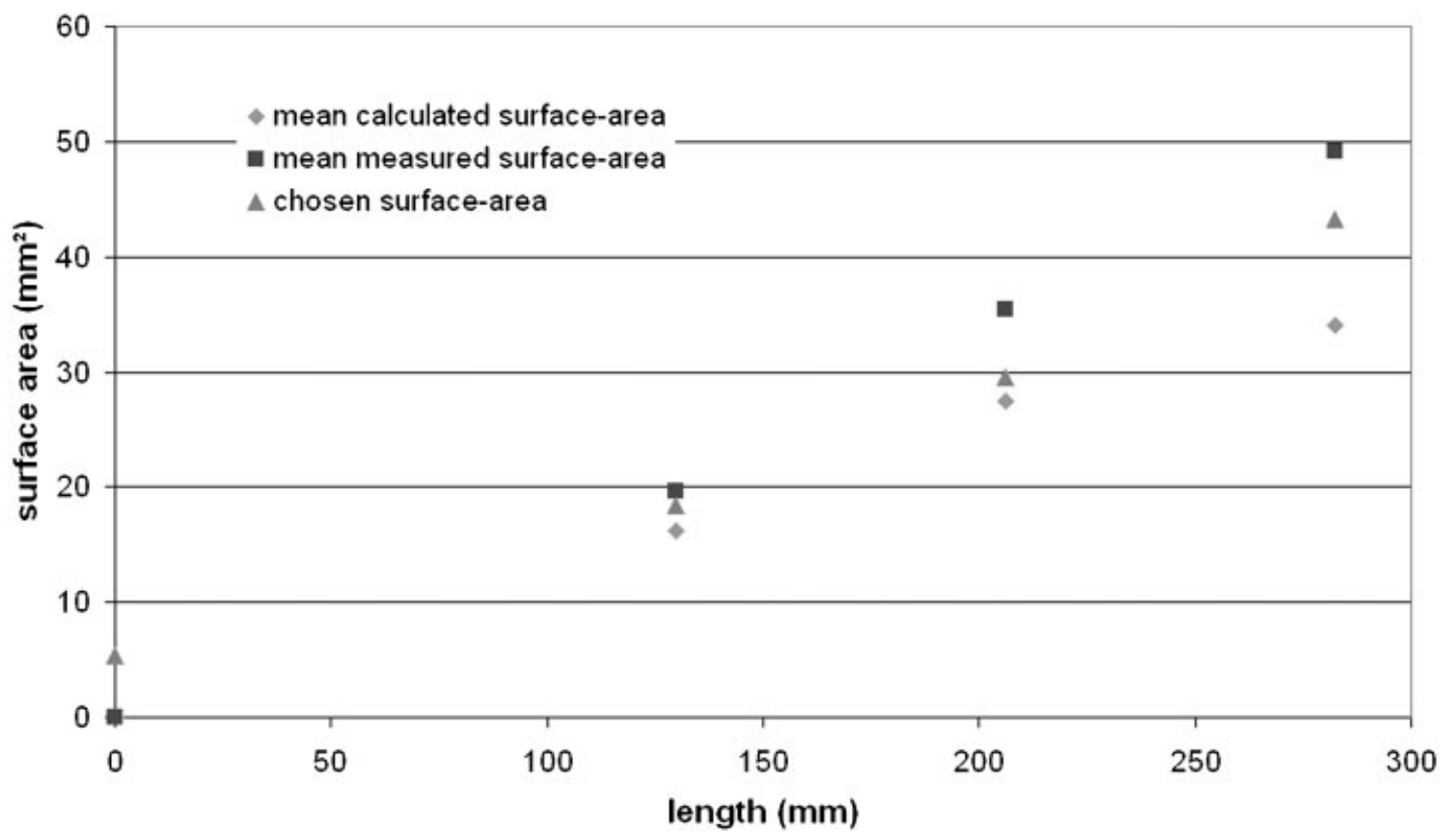

Fig. 3 Dimensions of the SSS (in-vivo measurements): $\bullet$ mean calculated surface area; mean measured surface area; $\boldsymbol{\Delta}$, chosen surface area (more detail in Fig. 4) 

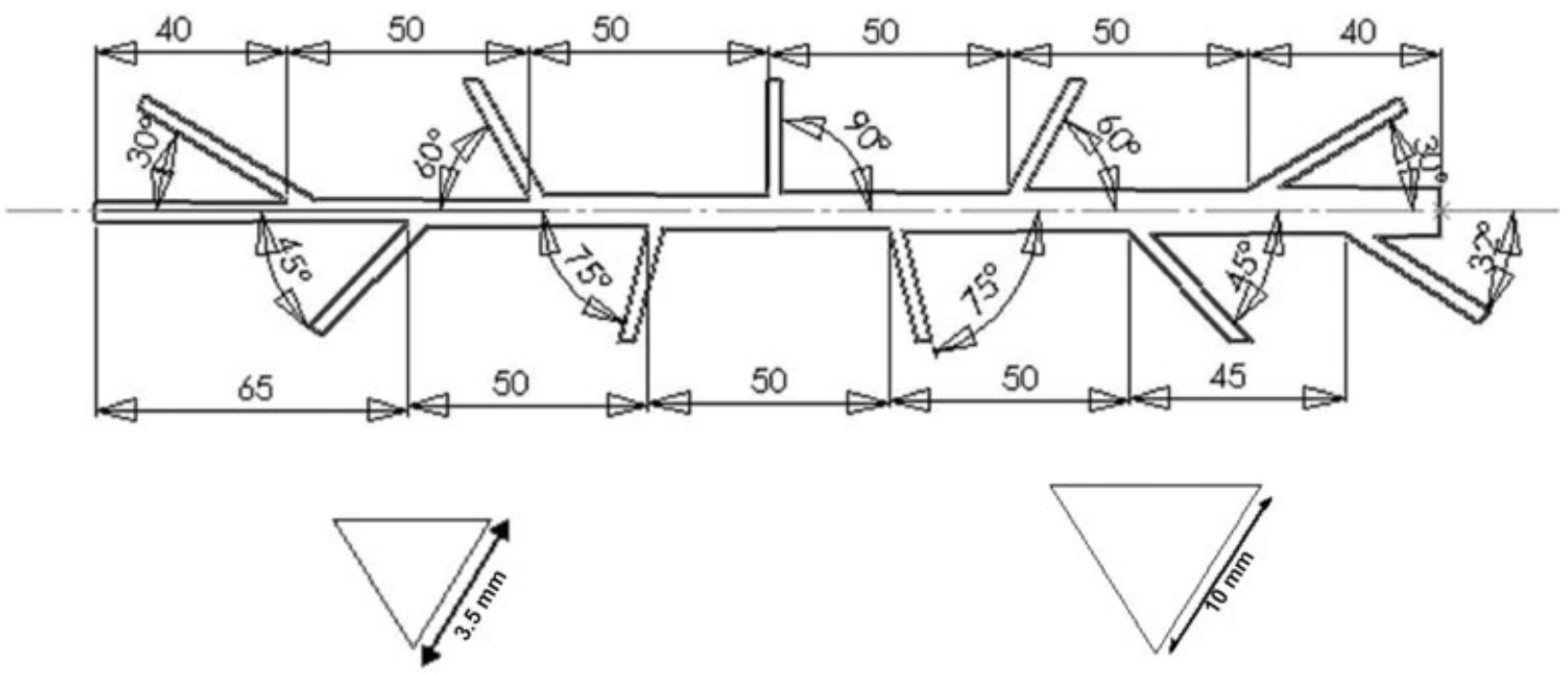

Fig. 4 Dimensions (mm) of the chosen SSS: detailed view of upstream (left) and downstream (right) cross-sections

of cerebral veins. A syringe pump with a constant inflow of $0.5 \mathrm{ml} / \mathrm{h}$ generated a steady state pressure in the vein and compensated the permanent leakage. The compliance curve in Fig. 5 was obtained with a syringe and a fluid-filled pressure transducer (type DT-XX, Becton Dickinson, Franklin Lakes, USA). Similar compliance tests were performed on different Penrose tubes in order to find the appropriate substitute material for the cortical veins. Finally, Penrose tubes of type 2 reference 473600 ( $8 \mathrm{~mm}$ diameter) were used. The compliance curve for this material is shown in Fig. 6. Ten Penrose tubes of $5.1 \mathrm{~cm}$ length yield the same compliance as 40 cortical veins in vivo (Fig. 2, D). As mentioned above, a windkessel provided the additional compliance to match the physiological compliance of the CSF system.

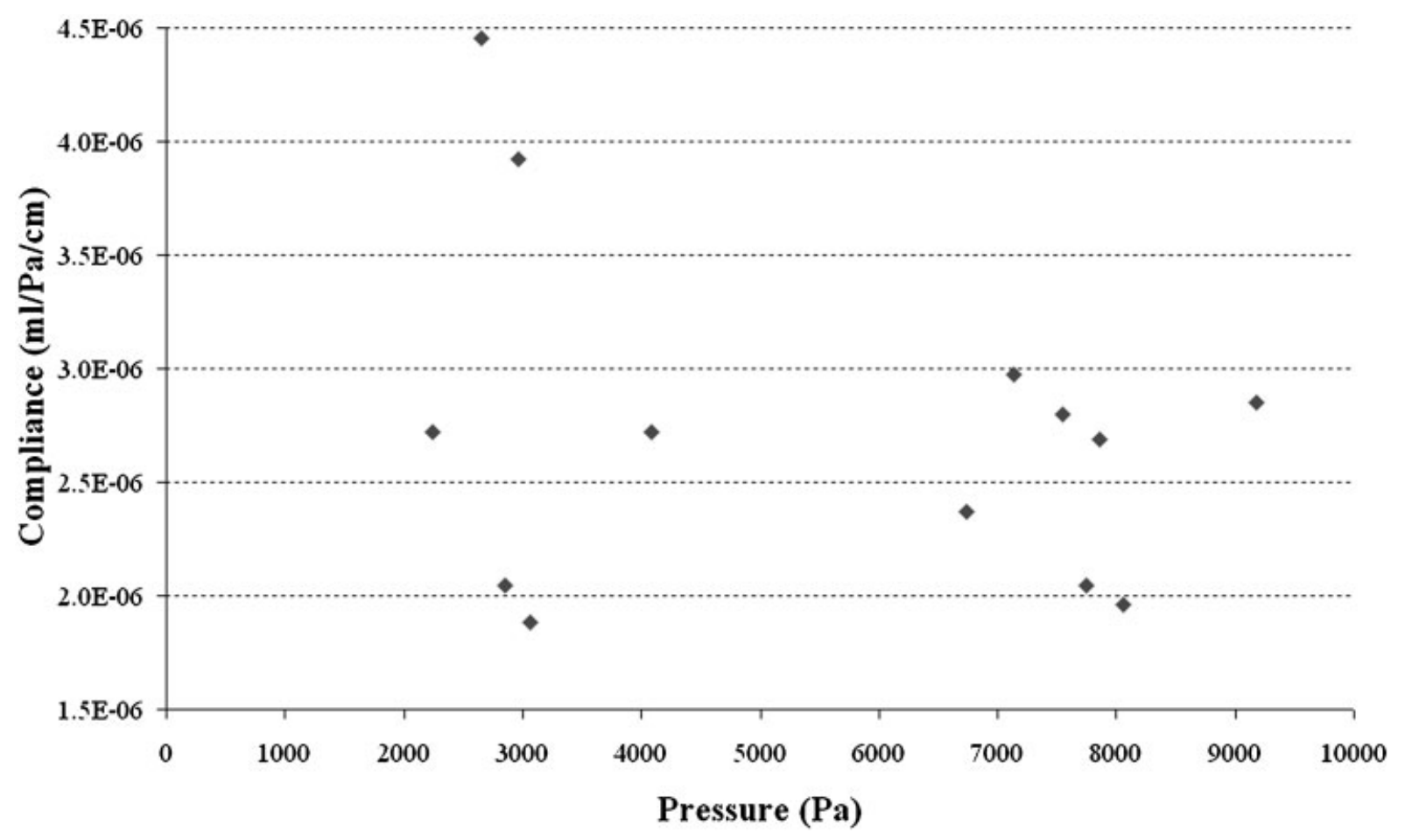

Fig. 5 Scatter plot of cortical vein compliance (in vivo) 


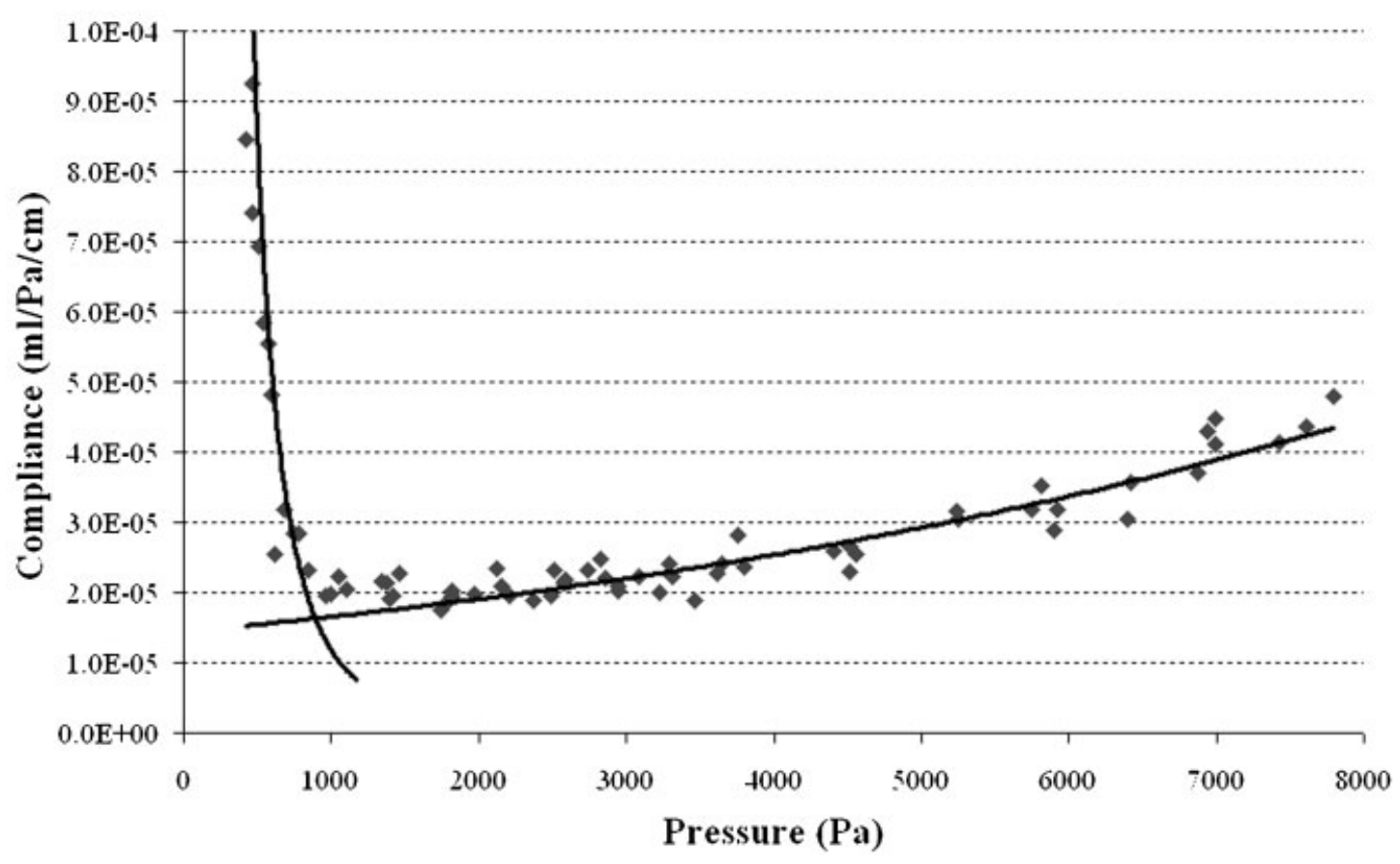

Fig. 6 Compliance curve for the Penrose tube (100-900 Pa: compliance, $4006.3 p^{-2.843}$ $\left.\left(R^{2}=0.8903\right)\right)\left(2000-8000\right.$ Pa: compliance, $\left.1.4 \times 10^{-5} \exp \left(10^{-4} p\right)\left(R^{2}=0.8906\right)\right)$

Blood is modelled by a 40 per cent glycerine- 60 per cent water mixture with a mass density of $1103 \mathrm{~kg} / \mathrm{m}^{3}$ and a dynamic viscosity of $3.75 \mathrm{mPa} \mathrm{s}$ at $25{ }^{\circ} \mathrm{C}$. A volumetric pump $(200 \mathrm{ml} / \mathrm{min}$ [15] $)$ brings this mixture into a distributor, where it is divided over ten rigid tubes (Fig. 2, E, F, and G). These tubes lead the mixture into the CSF reservoir where it flows into the SSS through the cortical veins, modelled by the Penrose tubes. The downstream pressure in the SSS is set by an adjustable-pressure reservoir which captures the glycerine-water mixture (Fig. 2, H).

In the CSF reservoir, the CSF surrounds the cortical veins and the modelled SSS. The shunt has an internal diameter of $1.1 \mathrm{~mm}$ and an external diameter of $2.2 \mathrm{~mm}$. To study an antegrade and a retrograde position, two entrances were made in the SSS at an angle of $45^{\circ}$ (Fig. 2, I). The antegrade shunt enters at $18 \mathrm{~cm}$ from the beginning of the SSS (upstream, smallest part). The shunt is placed $4 \mathrm{~cm}$ in the SSS so that the shunt tip is located $22 \mathrm{~cm}$ from the SSS beginning. For the retrograde set-up, the shunt was inserted at $20 \mathrm{~cm}$ and the shunt tip was at $16 \mathrm{~cm}$ from the beginning of the SSS.

During the tests, there was no extra CSF input in the system. The CSF pressure was raised to $3000 \mathrm{~Pa}$ and the pressure at the end of the SSS was fixed at $1000 \mathrm{~Pa}$. The CSF pressure, corresponding to the
ICP, and the pressure in the SSS were measured until they both stabilized. The pressure measured in the SSS is the static pressure in the SSS at the position of the shunt end. Since there is no CSF input, CSF flow through the shunt in the steady state condition will also be absent. This implies that the pressure in the CSF reservoir (ICP) is equal to the pressure in the shunt. Three tests for the retrograde set-up and three tests for the antegrade set-up were performed. Since small pressure differences are expected, differential water columns were used to perform pressure measurements.

To investigate the possibility of reflow of blood into the shunt, the pressure in the SSS was increased by $100 \mathrm{~Pa}$, starting from steady state conditions. This was achieved by raising the downstream pressure reservoir [8], mimicking the change from the upright to the lying position. Similarly, the ICP was lowered by $100 \mathrm{~Pa}$, to mimic a lumbar puncture.

\subsection{Numerical model}

The numerical model focuses mainly on the flow in the SSS itself. For this reason, only the inlet veins, the SSS, and an outflow vein were constructed in a three-dimensional model with exactly the same geometry as the experimental model. Flow and pressure boundary conditions were equal to those 
in the experimental model, and again both the antegrade and the retrograde configurations were studied. Fluent 6.2 (Fluent Inc., Sheffield, UK) was used to solve numerically the steady state NavierStokes equations. The complete model consists of over $1.14 \times 10^{6}$ finite volume mesh elements. The veins and the shunt were meshed with a successive ratio of 1.015 towards the outflow in the SSS. The SSS was filled with approximately 800000 triangular cells. As in the experimental model, the pressure differences between shunt and SSS at the end of the shunt were studied.

Additionally, a parameter study was conducted. A different blood flowrate in the SSS, $400 \mathrm{ml} /$ min instead of $200 \mathrm{ml} / \mathrm{min}$, was simulated in both the antegrade and the retrograde positions. The influence of the shunt position more upstream or downstream from the SSS was assessed by studying the velocity profile along the longitudinal axis of the SSS.

\section{RESULTS}

\subsection{Experimental model}

In the antegrade position, the static stabilization pressure in the CSF reservoir (ICP) is $3.3 \pm 2.5 \mathrm{~Pa}$ below the pressure in the SSS; this is the static pressure in the SSS at the position of the shunt end. In the retrograde set-up, the steady state ICP is $16.7 \pm 2.5 \mathrm{~Pa}$ higher than the static pressure in the SSS. Figure 7 shows the result of one test with the antegrade shunt and one test with the retrograde shunt.

While manipulating the model and increasing the SSS pressure (by $\left.100 \mathrm{~Pa}\left(1 \mathrm{cmH}_{2} \mathrm{O}\right)\right)$, simulating a person changing from upright to lying position [8], temporary reflow was observed until the pressure equilibrium was re-established. The same phenomenon of temporary reflow occurred when lowering the ICP, simulating a lumbar puncture $(100 \mathrm{~Pa})$.
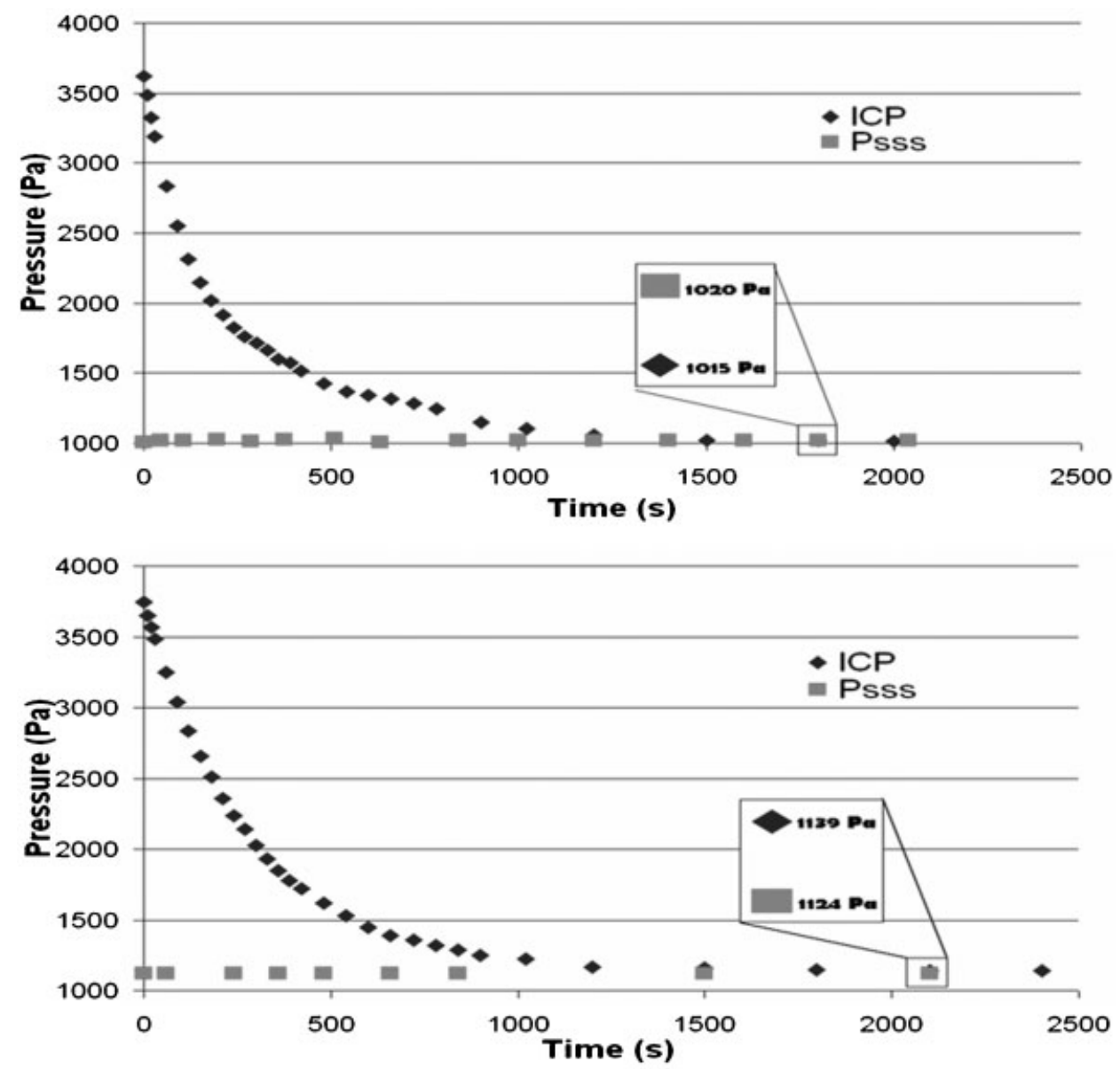

Fig. 7 Steady state static pressure: ICP and sinus pressure $P_{\mathrm{sss}}$ at the shunt tip: upper diagram, antegrade shunt; lower diagram, retrograde shunt 


\subsection{Numerical model}

In the antegrade numerical model, the ICP was $2 \mathrm{~Pa}$ lower than the static pressure in the SSS. With the shunt in retrograde position, the ICP was $13 \mathrm{~Pa}$ higher than in the SSS. Figure 8 shows the static pressure (Pa) in the SSS at the end of the shunt in both the antegrade and the retrograde positions.

When doubling the blood flowrate in the SSS ( $400 \mathrm{ml} / \mathrm{min})$, the pressure difference in the antegrade model remained almost constant. In the retrograde position, the pressure difference increased to $51 \mathrm{~Pa}$. The results are summarized in Table 1.

The maximum velocity profile in a sagittal crosssection of the SSS is shown in Fig. 9. The maximum velocity rises in the first $10 \mathrm{~cm}$ of the SSS and remains almost constant in the last $18 \mathrm{~cm}$.

\section{DISCUSSION}

As can be seen in Fig. 8, the steady state static pressure in the retrograde shunt is higher than in the
SSS. The total pressures in the SSS at the location of the shunt and inside the shunt are equal. Since there is no flow and consequently no dynamic pressure difference in the shunt, the static pressure in the shunt is equal to the total pressure in the SSS. As such, the static pressure in the shunt exceeds the static pressure in the SSS by the dynamic pressure in the SSS [7]. In other words, SSS blood flow stagnation at the shunt tip causes the static pressure in the shunt to rise by the amount of the dynamic pressure in the SSS, according to Bernoulli's principle: $\Delta p=\rho$ $(\Delta v)^{2} / 2$, with $\rho$ the density $\left(\mathrm{kg} / \mathrm{m}^{3}\right)$ and $v$ the velocity $(\mathrm{m} / \mathrm{s})$. This effect was called the 'impact effect' by ElShafei and El-Shafei [7].

At the position of the shunt tip, the blood velocity in the SSS is about $0.15 \mathrm{~m} / \mathrm{s}$. The CSF velocity in the shunt for steady state conditions is $0 \mathrm{~m} / \mathrm{s}$, corresponding to a pressure difference of $12.5 \mathrm{~Pa}$. This is comparable with the experimental and numerical results: $16.7 \mathrm{~Pa}$ and $13 \mathrm{~Pa}$ respectively.

In the antegrade position, it can be theoretically expected that the ICP is lower than the static pressure in the SSS. El-Shafei and El-Shafei [7] described this as a 'wake effect'. Bernoulli's principle

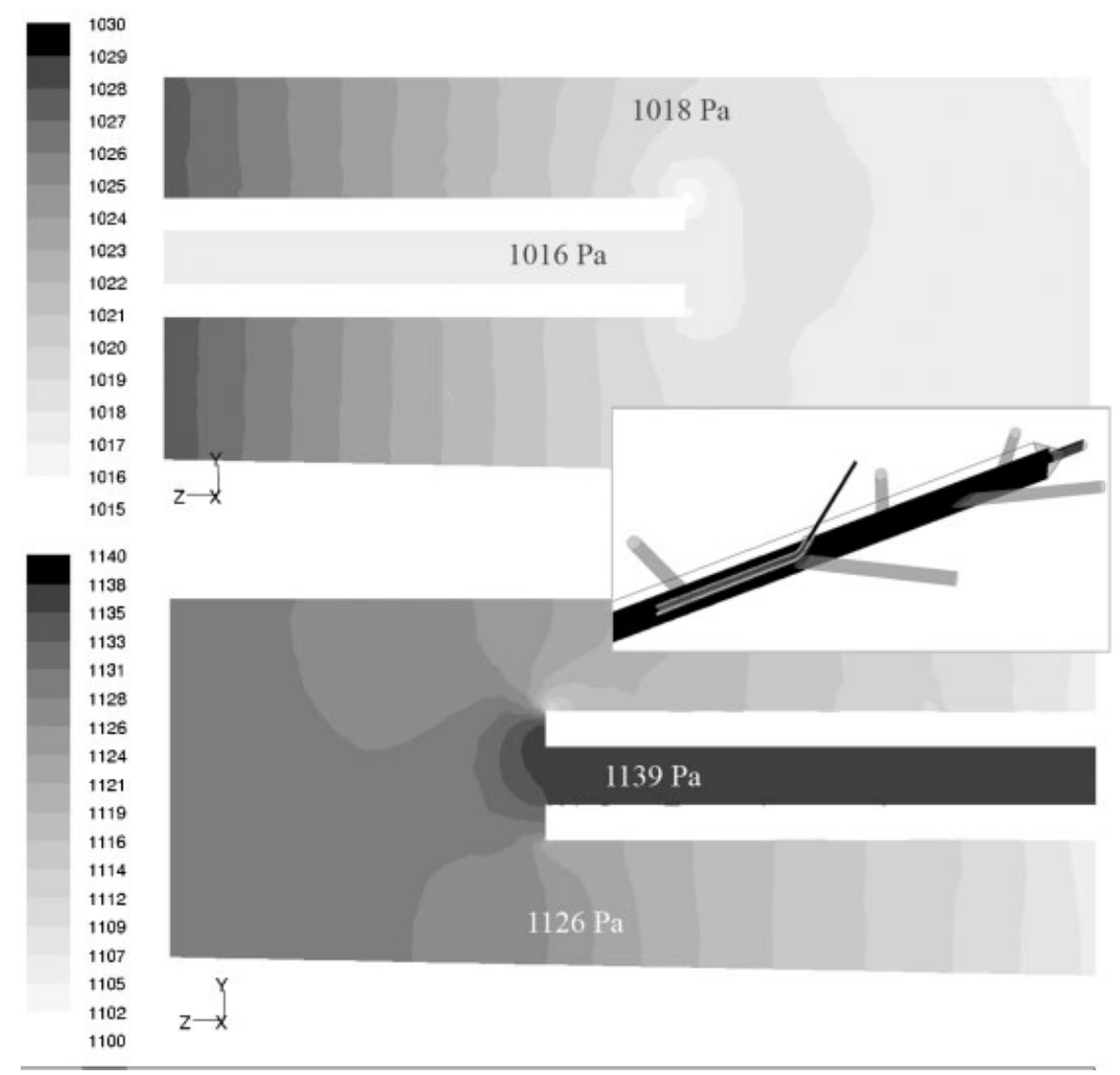

Fig. 8 Numerical steady state static pressure $(\mathrm{Pa})$ in the longitudinal plane: upper diagram, antegrade shunt in the SSS; lower diagram, retrograde shunt in the SSS 
Table 1 Difference between the ICP and pressure $P_{\text {sss }}$ in the SSS at the shunt tip for both the antegrade set-up and the retrograde set-up: experimental and numerical results

\begin{tabular}{llll}
\hline & \multicolumn{3}{c}{ Difference $(\mathrm{Pa})$ between the ICP and $P_{\mathrm{sss}}$} \\
\cline { 2 - 4 } Set-up & Experimental & Numerical & $\begin{array}{l}\text { Numerical } \\
\text { (SSS double flow) }\end{array}$ \\
\hline Antegrade & $-3.3 \pm 2.5$ & -2 & -2 \\
Retrograde & $16.7 \pm 2.5$ & 13 & 51 \\
\hline
\end{tabular}

cannot be used here, which implies that no result can be calculated in an analytical way. The experimental and numerical results show that the values obtained for the pressure in the CSF reservoir are 3.3 $\mathrm{Pa}$ and $3 \mathrm{~Pa}$ respectively lower than in the SSS. The lower CSF pressure confirms the existence of the wake effect.

When doubling the SSS blood flowrate, the difference between the ICP and the SSS in the retrograde model changed quadratically with the blood flow velocity. This corresponds to the quadratic relationship between the pressure difference and blood flow velocity in the retrograde position according to Bernoulli's principle. In the antegrade position, the pressure difference remained constant, illustrating that an increased SSS flowrate does not trigger overdrainage.

In the numerical parameter study, the influence of the shunt position was studied in detail. Conclusions were made based on the velocity profile. When advancing more downstream in the SSS, the blood flowrate is higher and the surface area becomes larger. In the first $10 \mathrm{~cm}$ of the SSS, the velocity increased from $0.04 \mathrm{~m} / \mathrm{s}$ to $0.15 \mathrm{~m} / \mathrm{s}$. In the last $18 \mathrm{~cm}$, however, the mean velocity in the SSS remained almost constant.

Since the blood velocity has no influence on the difference between the ICP and the SSS pressure in the antegrade set-up (see increased SSS flow), it is clear that shunt position has no influence either.

In the retrograde set-up, however, the impact pressure will rise as the shunt is positioned more downstream in the first $10 \mathrm{~cm}$ of the SSS, to reach a more constant value in the last $18 \mathrm{~cm}$. Therefore it seems recommendable for clinical practice to insert the shunt in the last $18 \mathrm{~cm}$ of the SSS (downstream part of the SSS).

In the first experiments, no reflow was observed in either numerical or experimental tests. As mentioned above, temporary reflow was only observed while rapidly lowering the ICP (mimicking lumbar puncture) or increasing the SSS pressure. Interestingly, recent clinical studies did not observe any temporary reflow $[\mathbf{1 6}]$. The reason for this discrepancy could be the constant CSF flow through the shunt in in-vivo conditions. The physiological CSF production ratio is $0.35 \mathrm{ml} / \mathrm{min}$, while there was no CSF production in the experiments. The difference between the pressure in the SSS at the position of the shunt tip and the pressure in the shunt tip will be approximately the same when CSF production is incorporated as without CSF production. Owing to the very low CSF flowrates the change in impact or wake effect is negligible. However, the difference

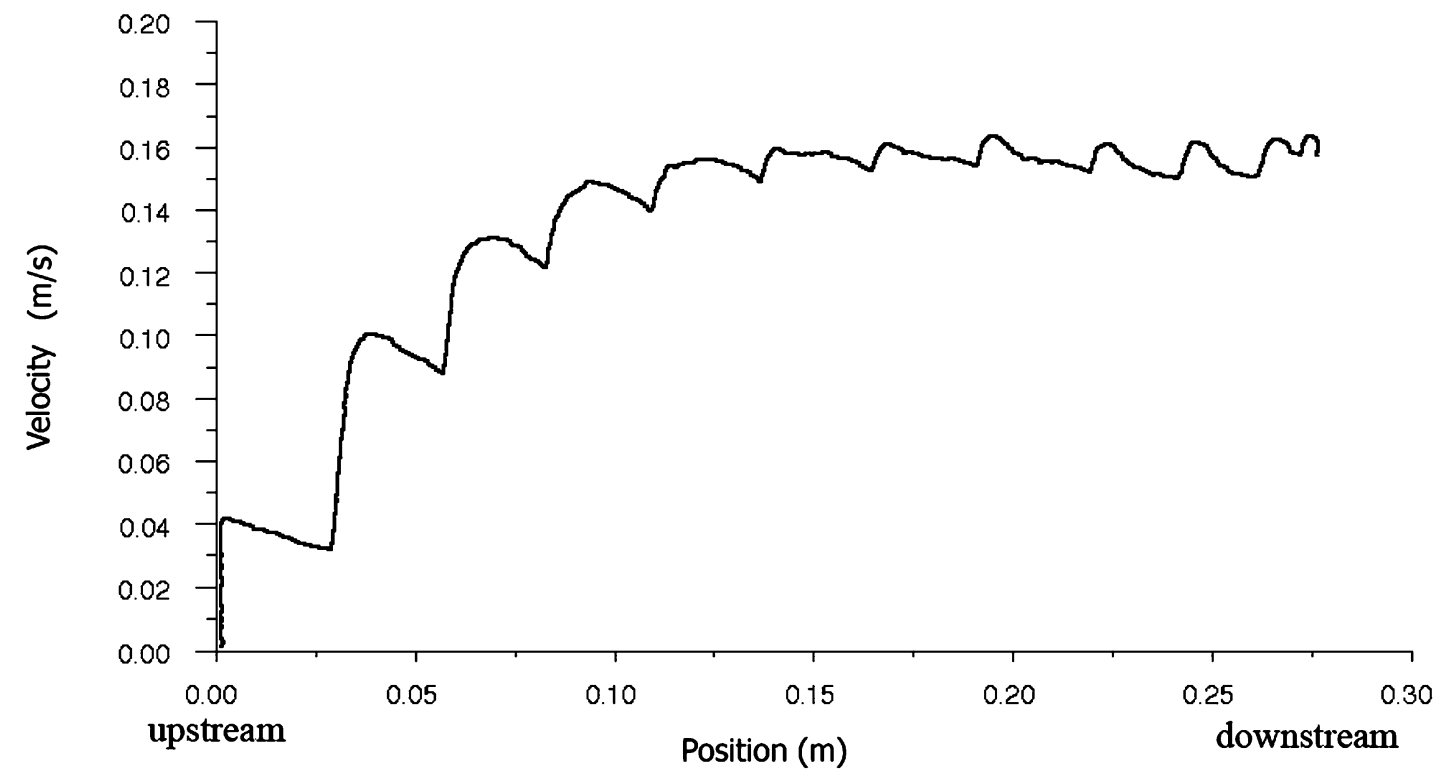

Fig. 9 Maximal velocity distribution in the SSS (sagittal plane) 
between the pressure in the CSF reservoir (ICP) and the pressure in the SSS at the shunt tip is increased by the pressure loss in the shunt. According to Poiseuille's law, this pressure drop can amount to 50-100 Pa, possibly explaining the higher capacity to withstand the natural pressure changes without reflow in clinical studies [16]. According to this study, however, an overall no-reflow configuration is not possible without any valve or other mechanical device.

During the compliance measurement of the cortical vein, only one cortical vein with $2 \mathrm{~cm}$ length was measured. The use of this limited length can be discussed and is one of the limitations of the study. However, as the total imposed cerebral compliance was obtained from the literature [9], Fig. 7 is expected to change only slightly when a more validated vein compliance is used.

A second limitation is the blood flow distribution over the cortical veins. The present authors did not find any relevant data in literature which describes the blood flow distribution along the human SSS. In both the experimental and the numerical models, the blood flow of $200 \mathrm{ml} / \mathrm{min}$ was distributed uniformly over the veins. This choice can lead to a velocity field in the modelled SSS that is different from the velocity field in the human SSS. Therefore, the recommendation of this study to insert the retrograde shunt in the last $18 \mathrm{~cm}$ of the SSS might have to be slightly adjusted as this distance depends on the inflow distribution in the SSS.

\section{CONCLUSION}

This study yields a predictive model to investigate VS shunting as a treatment for malresorptive hydrocephalus. The experimental measurements yield validation for the newly developed numerical model.

According to the experimental and numerical tests, shunting the cerebral ventricles to the SSS is possible without permanent reflow, both in the antegrade and in the retrograde configurations. Nevertheless, in order to apply this technique clinically and given the small pressure differences, the fact that a pressure change in the system (e.g. sitting upright against lying down, or lumbar puncture) can cause a temporary reflow of blood in the shunt should be taken into consideration. According to El-Shafei and El-Shafei [16] a simple unidirectional check valve with the least opening pressure can prevent this temporary blood reflow and should be implemented. The best clinical results are expected for a shunt positioned in the most downstream half of the SSS.

\section{REFERENCES}

1 Browd, S. R., Ragel, B. T., Gottfried, O. N., and Kestle, J. R. Failure of cerebrospinal fluid shunts: part I: obstruction and mechanical failure. Pediat. Neurology, 2006, 34(2), 83-92.

2 Browd, S. R., Gottfried, O. N., Ragel, B. T., and Kestle, J. R. Failure of cerebrospinal fluid shunts: part II: overdrainage, loculation, and abdominal complications. Pediat. Neurology, 2006, 34(3), 171-176.

3 Drake, J. M., Kestle, J. R., and Tuli, S. CSF shunts 50 years on-past, present and future. Childs Nerv. System, 2000, 16(10-11), 800-804.

4 Naradzay, J. F., Browne, B. J., Rolnick, M. A., and Doherty, R. J. Cerebral ventricular shunts. J. Emergency Medicine, 1999, 17(2), 311-322.

5 Pudenz, R. H. and Foltz, E. L. Hydrocephalus: overdrainage by ventricular shunts. A review and recommendations. Surg. Neurology, 1991, 35(3), 200-212.

6 El-Shafei, I. L. Ventriculovenous shunt against the direction of blood flow: a new approach for shunting the cerebrospinal fluid to the venous circulation. Childs Nerv. System, 1985, 1(4), 200-207.

7 El-Shafei, I. L. and El-Shafei, H. I. The retrograde ventriculosinus shunt: concept and technique for treatment of hydrocephalus by shunting the cerebrospinal fluid to the superior sagittal sinus against the direction of blood flow. Preliminary report. Childs Nerv. System, 2001, 17(8), 457-465; discussion, 466.

8 El-Shafei, I. L. and El-Rifaii, M. A. Ventriculojugular shunt against the direction of blood flow. I. Role of the internal jugular vein as an antisiphonage device. Childs Nerv. System, 1987, 3(5), 282-284.

9 Marmarou, A., Shulman, K., and LaMorgese, J. Compartmental analysis of compliance and outflow resistance of the cerebrospinal fluid system. $J$. Neurosurg., 1975, 43(5), 523-534.

10 Lang, J. Kopf, Teil a Übergeordnete Systeme, 2000 (Springer-Verlag, Berlin).

11 Lazorthes, G., Gouazé, A., and Solamon, G. Vascularisation et circulation de l'encéphale, tome premier anatomie descriptive et fonctionelle, 1975 (Masson, Paris).

12 Testut, L. Traité d'anatomie humaine, 1930 (Gaston, Paris).

13 Cirovic, S., Walsh, C., and Fraser, W. D. Mathematical study of the role of non-linear venous compliance in the cranial volume-pressure test. Med. Biol. Engng Computing, 2003, 41(5), 579-588. 
14 Ursino, M. and Lodi, C. A. A simple mathematical model of the interaction between intracranial pressure and cerebral hemodynamics. J. Appl. Physiology, 1997, 82(4), 1256-1269.

15 Kim, J., Thacker, N. A., Bromeley, P. A., and Jackson, A. Emperical validation of cerebrospinal fluid pulsatility model. Tina Memo 2004-011, 2004, p. 15.

16 El Shafei, I. L. and El Shafei, H. I. The retrograde ventriculo-sinus shunt (El Shafei RVS shunt). Rationale, evolution, surgical technique and long-term results. Pediat. Neurosurg., 2005, 41(6), 305-317.

\section{APPENDIX}

\section{Notation}

CSF

ICP

SSS

$v$

VS

$\Delta p$

$\rho$ cerebrospinal fluid intracranial pressure superior sagittal sinus velocity ventriculosinus

pressure difference density 